\title{
Lay concepts of source likeability, trustworthiness, expertise, and power: A prototype analysis
}

\author{
Laura E. Wallace $^{1}$ (D) Kay A. Simon ${ }^{2} \cdot$ Duane T. Wegener $^{1}$ \\ Accepted: 2 September 2020 / Published online: 1 October 2020 \\ (C) The Psychonomic Society, Inc. 2020
}

\begin{abstract}
Previous research on persuasion has used researcher-generated exemplars to manipulate source characteristics such as likeability, trustworthiness, expertise, or power. This approach has been fruitful, but it relies to some degree on an overlap between researcher understanding of these variables and lay understanding of these variables. Additionally, these exemplar manipulations may have unintentionally affected multiple characteristics and may be limited to certain topics or time periods. In the current work, we sought to provide persuasion researchers with a methodological tool to increase construct and potentially external validity by conducting a prototype analysis of the four traditional source characteristics: likeability, trustworthiness, expertise, and power. This bottom-up approach provided insight into the ways in which recipients perceive sources and allowed us to examine relations between the characteristics. Moving forward, a bottom-up understanding of source characteristics will allow researchers to more effectively develop manipulations that might transcend time and topic as well as isolate their effects to the intended source characteristic.
\end{abstract}

Keywords Persuasion $\cdot$ Sources $\cdot$ Likeability $\cdot$ Trustworthiness $\cdot$ Expertise $\cdot$ Power

When people receive persuasive messages, they often attend to features of the source providing the message. They can pay attention to whether a salesperson is likeability, a corporation providing an advertisement is truthful, an advocate is knowledgeable about her cause, or a political candidate has power to enact the policies she endorses. The way they perceive these sources could ultimately determine the extent to which they are persuaded.

Indeed, since the beginning of persuasion science, researchers have identified source characteristics as one of the core factors that affect persuasion (e.g., Hovland, Janis, and Kelley 1953; Kelman and Hovland 1953). In this early work, Kelman (1958) taxonomized source perceptions into attractiveness (likeability), trustworthiness, expertise, and power.

Electronic supplementary material The online version of this article (https://doi.org/10.3758/s13428-020-01478-1) contains supplementary material, which is available to authorized users.

Laura E. Wallace

lwalla2@gmu.edu

1 Department of Psychology, The Ohio State University, 1835 Neil Avenue, Columbus, OH 43210, USA

2 Department of Psychology, University of Kentucky, Lexington, KY, USA
Kelman discussed attractiveness as the extent to which other people liked the source. Some manipulations of source attractiveness have used physical characteristics (e.g., Snyder and Rothbart, 1971), whereas others have used qualities that might make the source likeability or dislikeability in other ways such as celebrity versus average-joe status, politeness versus impoliteness, or compliments versus insults of the message recipient or their group (e.g., Abelson and Miller, 1967; Petty, Cacioppo, and Schumann, 1983; Petty, Wegener, and White, 1998; Zimbardo et al., 1965). Research has suggested that even when manipulations of the source's appearance have been used, the effects on persuasion were mediated by a more general liking for the source (see Chaiken, 1986). Further, Kelman (1958) conceptualized trustworthiness and expertise as two components of source credibility. He discussed trustworthiness as the source's intent to communicate truthfully and expertise as the source's knowledge or training on the relevant topic. Finally, he defined power as the ability of a communicator to control positive and negative sanctions for the receiver and to know whether the recipient accepted the source's position. Since identification of these key source characteristics, a wealth of research has documented that these characteristics can indeed influence persuasion, and can do so through multiple different processes (Chaiken, Liberman, \& Eagly, 1989; Petty \& Cacioppo, 1986). 
In all this previous work, researchers have employed researcher-generated manipulations of each characteristic. Typically, these manipulations have been based on exemplars that researchers believed capture either the positive or negative end of a continuum representing one of the characteristics. For example, in a study on the effects of source trustworthiness, Priester and Petty (2003) provided participants with a message advertising rollerblades and used Nancy Kerrigan as the source in the trustworthy condition and Tonya Harding in the untrustworthy condition. Both Kerrigan and Harding were famous ice-skating stars from the United States at the time the study was run. The US-based participants used in the sample would have been aware of a controversy in which Harding's ex-husband orchestrated an attack on Kerrigan so that she would not be able to compete against Harding. At the time, Harding's denials and perceived lack of truthfulness were assumed to make Harding seem untrustworthy. Similarly, in a study on the effects of source expertise, (Petty, Cacioppo, \& Goldman, 1981) provided participants with a message in support of implementing senior comprehensive exams at a university. In the "high expertise" condition, participants were informed that the message came from the Carnegie Commission on Higher Education chaired by a professor of education at Princeton University; in the "low expertise" condition, the message came from a class at a local high school.

This reliance on researcher-generated exemplars could have introduced threats to construct and perhaps external validity. Although this previous work has clearly demonstrated that their operationalizations can influence the intended source characteristic (via manipulation checks) and persuasion, relatively little is known about the ways in which the average person thinks about each of the source characteristics and the defining features that would correspond with each. If researcher and participant understanding of these characteristics differ, it could introduce a construct validity issue. That is, researchers may conclude that any observed effects were due to the wrong construct. An understanding of the lay conceptualization of each source characteristic would not only provide insight into the ways in which the average person views message sources, but it would also allow researchers to develop manipulations that would reflect people's lay conceptions of the source constructs, and thus potentially increase construct validity. Further, the exemplars used in previous work are likely to be vulnerable to change over time or topic. The use of Nancy Kerrigan and Tonya Harding as sources is perhaps particularly emblematic of this, as many readers of the current article may not have known who they were without explanation. As such, a bottomup approach to source characteristics could move toward identifying the key features in lay perceptions that capture the essence of each characteristic and would be needed in new manipulations and for new topics.

Additionally, very little is known about possible interrelations between source characteristics, resulting in other potential construct validity threats and perhaps even external (generalizability) threats. In previous work, researchers typically focused on the effects of one source characteristic at a time. However, the manipulations they used might have influenced more than one, and maybe several, source characteristics. For example, in the research by Petty et al. (1981) described above, manipulation checks confirmed that participants perceived the Carnegie Commission as more expert than high school students. However, researchers did not measure other source characteristics to examine if the manipulation would also have effects on those, introducing potential construct validity concerns. One might imagine that participants would view the Carnegie Commission as more powerful, since they might be able to influence the university, whereas high school students would not. Participants might also like the Carnegie Commission more if the high school students are perceived as immature or angsty. If this manipulation and others like it had effects on other source characteristics, it would be unclear whether the effects on persuasion were due to expertise per se rather than one of the other characteristics or their combination. Understanding the prototype of each source characteristic would allow for an examination of conceptual overlap between the characteristics, which might allow researchers to create manipulations that more uniquely affect each characteristic. At the very least, it would allow researchers to determine when they might need to control for other perceived source characteristics. Issues related to construct validity might also have implications for external validity or generalizability. That is, effects of different source characteristics might be moderated by different variables (i.e., might generalize to particular situations or respondents but not to others). If so, then manipulations aimed at one source perception that instead influence another might lead to effects that are moderated in ways that are difficult for researchers to anticipate or understand unless those alternative source perceptions are taken into account.

As such, the goal of the current research is to better understand the lay conceptions that people have of the four originally identified source characteristics. By having participants generate descriptions of the characteristics, we were able to develop prototypes of each, providing bottom-up insight into the core features of the characteristics as understood by lay perceivers. Ultimately, our hope is that these prototypes will serve as a methodological tool to help researchers develop manipulations that better capture the core elements of each source characteristic. These new manipulations could apply across time and topic (especially if using the features themselves, rather than an exemplar thought to embody those features) 
and isolate the source characteristic(s) responsible for persuasion effects.

\section{Study 1: Elicitation}

Because the purpose of this research was to develop prototypes for each source characteristic, in the elicitation phase of research, participants generated descriptions and defining features of each source characteristic. All of the studies in the manuscript were approved by the Ohio State Office of Responsible Research Practices and conform to recognized standards. For all studies, participants provided their consent prior to their inclusion in the study.

\section{Method}

Participants One hundred and twenty-two participants (52.50\% female; $82 \%$ White, $7.2 \%$ Black, $4.1 \%$ Asian, .8\% Native American, $4.9 \%$ Multiracial, $8.9 \%$ Hispanic or Latino; Mage $=36.93$, SDage $=14.26$ ) recruited from Amazon's Mechanical Turk participated in this study. Participants were paid $\$ 0.50$, and the median completion time was about seven minutes.

Design Participants generated descriptions of the core source characteristics: likeability, power, trustworthiness, and expertise $^{1}$. We were concerned that participants would get fatigued if asked to generate words and phrases for all characteristics. As such, each participant only responded to questions regarding three of the characteristics. Additionally, they only responded to either the positive or negative version of each characteristic (e.g., expert versus inexpert; a between-subjects factor). We randomized the combination and order of characteristics to control for these extraneous effects.

Procedure Participants read that the purpose of the study was to examine perceptions of others trying to persuade. They were told that they would be asked to write a series of six short essays. Then they were instructed to imagine that someone was trying to persuade them, and the persuader was described as possessing one of the characteristics. They were

\footnotetext{
${ }^{1}$ In this study, participants also generated descriptions for an additional source characteristic: bias, which recent work has identified as an independent source perception (Wallace, Wegener, and Petty, 2020a, b). However, for the additional studies reported in this manuscript, we decided to focus on the four traditional source characteristics. Therefore, the bias-related elicitations are not reported here. For interested readers, a quick summary of this recent work follows: participants think of perceived bias as a motivation to hold a particular position. Like these other source perceptions, perceived bias tends to be correlated with each of these other perceptions but can have independent influences on persuasion. Further, people can infer source bias without necessarily making inferences about other perceptions, and source bias can, at times, have different consequences than other perceptions (Wallace, Wegener, and Petty, 2020a, b)
}

asked to define the characteristic and describe how they would know that the person possessed the given characteristic. Participants completed the same questions for two other characteristics. Prior to answering the questions, they were informed that they would have a minimum of 30 seconds and a maximum of a minute and a half to write each essay. We programmed the study so that the two questions for each characteristic were on the same page. Participants could not advance to the next screen until 60 seconds had passed (30 seconds for each question) and were automatically advanced to the next screen after 180 seconds (a minute and a half for each question). We specified a window of time that people must and could spend on each essay because we wanted to give participants sufficient time to be thoughtful in their answers, but we did not want participants to write long essays about each word (so the generated content would primarily reflect the core features of the source characteristic). Finally, participants responded to demographic questions and were debriefed about the purpose of the study.

Measures Participants were asked two questions about each of the three characteristics that they were assigned. First each participant was asked, "Imagine a situation in which a person is trying to persuade you and they are [CHARACTERISTIC (e.g., expert)]. How would you define [CHARACTERISTIC]?" Next, participants responded to "How would you know they are [CHARACTERISTIC]? What are the clues you would use to determine that this person is [CHARACTERISTIC]?" The first question was intended to assess people's definitions of each characteristic. The second and third questions were intended to assess the key features of the source characteristic and the cues that might lead people to infer that a source had a particular characteristic. We asked people these questions to generate a wide range of responses that might come to mind across contexts for each characteristic. Additionally, we wanted to give participants the opportunity to write phrases or sentences, rather than solely words. Words by themselves are more likely to be ambiguous in meaning, so giving participants the opportunity to write sentences and phrases provided additional opportunities to clarify their answers.

\section{Results}

A member of the research team extracted the words and phrases (descriptions) that participants used for each characteristic. We tried to be as inclusive as possible, including all the relevant descriptions that the participants generated. We only excluded responses from individuals who seemed as if they did not understand the task. Tables 1,2, 3, and 4 include the lists of descriptions we included as "prototypical" based on additional exclusions we made in Study 2. As explained in detail later, in Study 2, participants rated each of the 
Table 1 Informativeness ratings and the number of participants who generated the description for each (dis)likeability description

\begin{tabular}{|c|c|c|c|c|c|c|c|}
\hline \multicolumn{4}{|l|}{ Likeability } & \multicolumn{4}{|l|}{ Dislikeability } \\
\hline Description & $M$ & $S D$ & \# generating & Description & $M$ & $S D$ & \# generating \\
\hline Caring & 6.74 & 1.00 & 2 & Rude & 7.23 & 1.13 & 9 \\
\hline Kind & 6.58 & 1.35 & 4 & Cruel & 7.30 & 1.35 & 1 \\
\hline Genuine & 6.56 & 1.44 & 3 & Arrogant & 6.79 & 1.54 & 3 \\
\hline Friendly & 6.56 & 1.40 & 8 & Racist & 6.67 & 1.86 & 1 \\
\hline Nice & 6.26 & 1.16 & 3 & Mean & 6.60 & 1.53 & 3 \\
\hline Pleasant & 6.37 & 1.25 & 2 & Discriminatory & 6.60 & 1.68 & 1 \\
\hline Sincere & 6.37 & 1.33 & 1 & Hostile & 6.53 & 1.58 & 1 \\
\hline Considerate & 6.23 & 1.00 & 1 & Manipulative & 6.56 & 1.76 & 1 \\
\hline Helpful & 6.30 & 1.08 & 1 & Abrasive & 6.56 & 1.68 & 2 \\
\hline Warm & 6.21 & 1.37 & 1 & Impolite & 6.49 & 1.42 & 2 \\
\hline Empathetic & 6.12 & 1.29 & 1 & Disrespectful & 6.42 & 1.53 & 2 \\
\hline Respectful & 6.05 & 1.51 & 1 & Intolerable & 6.49 & 1.45 & 1 \\
\hline Supportive & 5.98 & 1.22 & 1 & Repelling & 6.28 & 1.78 & 1 \\
\hline Welcoming & 5.91 & 1.13 & 1 & Conniving & 6.49 & 1.88 & 1 \\
\hline Amiable & 5.88 & 1.37 & 1 & Nasty & 6.35 & 1.69 & 1 \\
\hline Positive & 5.81 & 1.64 & 4 & Condescending & 6.30 & 1.60 & 2 \\
\hline Cooperative & 5.79 & 1.39 & 1 & Intolerant & 6.33 & 1.57 & 1 \\
\hline Generous & 5.67 & 1.30 & 1 & Unkind & 6.33 & 1.58 & 1 \\
\hline Humble & 5.84 & 1.23 & 1 & Offensive & 6.21 & 1.74 & 1 \\
\hline Unselfish & 5.63 & 1.72 & 1 & Sexist & 6.19 & 1.89 & 1 \\
\hline Non-threatening & 5.65 & 1.54 & 3 & Unfriendly & 6.12 & 1.65 & 3 \\
\hline Accommodating & 5.63 & 1.36 & 1 & Selfish & 6.16 & 1.72 & 1 \\
\hline Easy-going & 5.60 & 1.20 & 1 & Aggressive & 6.07 & 1.91 & 1 \\
\hline \multirow[t]{11}{*}{ Fun } & 5.49 & 1.91 & 1 & Uncaring & 6.02 & 1.54 & 1 \\
\hline & & & & Obnoxious & 5.86 & 1.91 & 1 \\
\hline & & & & Unpleasant & 5.81 & 1.65 & 2 \\
\hline & & & & Confrontational & 5.88 & 1.78 & 2 \\
\hline & & & & Pushy & 5.81 & 1.48 & 4 \\
\hline & & & & Negative & 5.67 & 2.01 & 1 \\
\hline & & & & Creepy & 5.67 & 2.03 & 1 \\
\hline & & & & Angry & 5.70 & 2.06 & 1 \\
\hline & & & & Forceful & 5.58 & 2.05 & 1 \\
\hline & & & & Uninviting & 5.58 & 1.67 & 1 \\
\hline & & & & Argumentative & 5.63 & 2.00 & 1 \\
\hline
\end{tabular}

descriptions according to how informative they were for identifying whether a source has a particular characteristic $(1=$ not at all informative, $8=$ extremely informative). For efficiency's sake, these informativeness ratings are also included in these tables. Tables with all the descriptions generated exist in the supplemental materials. Tables 1, 2, 3, and 4 also include the number of participants who mentioned each description. The raw data and code (where relevant) for conducting analyses in this paper is available at https://osf.io/4e6hy/?view_only= e28832c0a5bf4ed38671579070461 fd3.

\section{Studies 2a-d: Informativeness ratings}

Study 1 allowed us to generate several descriptions for each source characteristic. However, for some characteristics, the number of descriptions generated was quite large. Among those source characteristics with the highest number of generated descriptions, participants generated 57 for a dislikeability source, 41 for a likeability source, and 40 for a powerless source. We were most concerned with establishing which words capture the essence of each characteristic. As such, 
Table 2 Informativeness ratings and the number of participants who generated the description for each (un)trustworthy description

\begin{tabular}{|c|c|c|c|c|c|c|c|}
\hline \multicolumn{4}{|l|}{ Trustworthy } & \multicolumn{4}{|l|}{ Untrustworthy } \\
\hline Description & $M$ & $S D$ & \# generating & Description & $M$ & $S D$ & \# generating \\
\hline Honest & 7.29 & 0.94 & 14 & Deceitful & 7.45 & 1.04 & 1 \\
\hline Sincere & 6.76 & 1.38 & 3 & Dishonest & 7.34 & 1.26 & 4 \\
\hline Reliable & 6.60 & 1.37 & 9 & Scheming & 7.19 & 1.31 & 1 \\
\hline Dependable & 6.49 & 1.43 & 10 & Unreliable & 7.06 & 1.21 & 9 \\
\hline Believable & 6.40 & 1.74 & 1 & Shady & 6.96 & 1.46 & 1 \\
\hline \multirow[t]{10}{*}{ Reputable } & 6.41 & 1.40 & 1 & Undependable & 6.81 & 1.39 & 2 \\
\hline & & & & Unaccountable & 6.70 & 1.23 & 1 \\
\hline & & & & Insincere & 6.43 & 1.63 & 1 \\
\hline & & & & Inconsistent & 6.43 & 1.63 & 3 \\
\hline & & & & Fake & 6.45 & 1.85 & 1 \\
\hline & & & & Unbelievable & 6.47 & 1.86 & 1 \\
\hline & & & & Sketchy & 6.32 & 1.60 & 2 \\
\hline & & & & Shifty & 6.28 & 1.51 & 2 \\
\hline & & & & Withholding & 6.38 & 1.51 & 1 \\
\hline & & & & Artificial & 6.04 & 1.84 & 1 \\
\hline
\end{tabular}

Table 3 Informativeness ratings and the number of participants who generated the description for each (in)expert description

\begin{tabular}{|c|c|c|c|c|c|c|c|}
\hline \multicolumn{4}{|l|}{ Expert } & \multicolumn{4}{|l|}{ Inexpert } \\
\hline Description & $M$ & $S D$ & \# generating & Description & $M$ & $S D$ & \# generating \\
\hline Knowledgeable & 7.29 & 1.01 & 27 & Unqualified & 6.27 & 2.27 & 3 \\
\hline Experienced & 7.20 & 1.10 & 12 & Inexperienced & 6.24 & 1.98 & 12 \\
\hline Qualified & 7.02 & 1.19 & 7 & Unintelligent & 5.98 & 2.12 & 1 \\
\hline Mastered & 6.85 & 1.41 & 1 & Uninformed & 5.83 & 2.34 & 1 \\
\hline Skillful & 6.85 & 1.11 & 1 & Untrained & 5.83 & 2.31 & 1 \\
\hline Competent & 6.76 & 1.18 & 1 & Stupid & 5.49 & 2.28 & 1 \\
\hline Trained & 6.66 & 1.28 & 4 & & & & \\
\hline Practiced & 6.63 & 1.13 & 2 & & & & \\
\hline Informed & 6.59 & 1.40 & 1 & & & & \\
\hline Educated & 6.51 & 1.25 & 7 & & & & \\
\hline Professional & 6.51 & 1.25 & 1 & & & & \\
\hline Reputable & 6.49 & 1.12 & 2 & & & & \\
\hline Accomplished & 6.46 & 1.48 & 1 & & & & \\
\hline Smart & 6.41 & 1.41 & 1 & & & & \\
\hline Studious & 6.41 & 1.40 & 1 & & & & \\
\hline Proven & 6.37 & 1.46 & 1 & & & & \\
\hline Demonstrated & 6.22 & 1.54 & 1 & & & & \\
\hline Fluent & 6.17 & 1.61 & 1 & & & & \\
\hline Resourceful & 6.12 & 1.47 & 1 & & & & \\
\hline Trustworthy & 6.00 & 1.84 & 2 & & & & \\
\hline Confident & 5.93 & 1.92 & 4 & & & & \\
\hline Analytical & 5.90 & 1.48 & 1 & & & & \\
\hline Convincing & 5.73 & 1.70 & 1 & & & & \\
\hline Published & 5.66 & 1.70 & 1 & & & & \\
\hline Objective & 5.61 & 1.81 & 3 & & & & \\
\hline
\end{tabular}


Table 4 Informativeness ratings and the number of participants who generated the description for each power(less) description

\begin{tabular}{|c|c|c|c|c|c|c|c|}
\hline \multicolumn{4}{|l|}{ Powerful } & \multicolumn{4}{|l|}{ Powerless } \\
\hline Description & $M$ & $S D$ & \# generating & Description & $M$ & $S D$ & \# generating \\
\hline In charge & 7.08 & 1.05 & 1 & Dependent & 6.78 & 1.21 & 1 \\
\hline Influential & 6.98 & 1.14 & 9 & Helpless & 6.65 & 1.39 & 1 \\
\hline Dominant & 6.75 & 1.08 & 2 & No choice & 6.65 & 1.55 & 1 \\
\hline Assertive & 6.60 & 1.08 & 1 & Weak & 6.65 & 1.66 & 1 \\
\hline Persuasive & 6.58 & 1.01 & 3 & No free will & 6.63 & 1.69 & 1 \\
\hline Wealthy & 6.25 & 1.81 & 4 & No Say & 6.58 & 1.96 & 1 \\
\hline Charismatic & 6.13 & 1.54 & 1 & Lacking confidence & 6.50 & 1.45 & 1 \\
\hline Achieving & 6.08 & 1.37 & 1 & Ignored & 6.48 & 1.54 & 1 \\
\hline Strong & 6.00 & 1.45 & 3 & Defenseless & 6.45 & 1.58 & 1 \\
\hline Expert & 5.88 & 1.52 & 1 & Will-less & 6.38 & 1.69 & 1 \\
\hline Forceful & 5.78 & 1.79 & 1 & Unsuccessful & 6.33 & 1.49 & 1 \\
\hline Resourceful & 5.75 & 1.60 & 1 & No strength & 6.20 & 1.79 & 1 \\
\hline Causing fear & 5.73 & 1.75 & 1 & Ineffective & 6.13 & 1.73 & 1 \\
\hline \multirow[t]{5}{*}{ Demanding } & 5.70 & 1.59 & 1 & Unheard & 5.85 & 1.97 & 1 \\
\hline & & & & Hopeless & 5.75 & 1.81 & 1 \\
\hline & & & & Poor & 5.70 & 2.07 & 1 \\
\hline & & & & Clueless & 5.58 & 1.84 & 1 \\
\hline & & & & Not knowledgeable & 5.55 & 1.81 & 1 \\
\hline
\end{tabular}

we conducted a series of studies in which we asked participants the extent to which each generated description was informative in determining whether the person had the matching characteristic. For example, we asked how informative knowing someone was honest would be in determining the extent to which the source was trustworthy or untrustworthy. Conducting this set of data collections allowed us to condense each list to the words that were most informative for each characteristic. Because there were so many descriptions for each characteristic, we ran a separate study for each source characteristic so that each participant only responded to questions about one of the characteristics.

\section{Method}

We conducted four separate studies to examine the informativeness of each description - one for each of the traditional source characteristics - trustworthiness, expertise, likeability, and power.

Participants Each of the four studies was conducted on Mechanical Turk. We attempted to obtain roughly 40 responses for each. As such, the trustworthiness study had 54 participants ( 7 did not report demographics; of those who did report demographics, the sample was $68.1 \%$ women; $72.3 \%$ White, $6.4 \%$ Black, $10.6 \%$ Asian, $8.5 \%$ Multiracial; $4.3 \%$ Hispanic or Latino; Mage $=38.77$, SDage $=14.48)$. The expertise study had 41 participants $(51.2 \%$ female; $68.3 \%$ White, 19.5\% Black, 9.8\% Asian, 2.4\% Multiracial; $4.9 \%$ Hispanic or Latino; Mage $=39.73$. SDage $=13.96$ ). The power study had 40 participants (45\% female; $87.5 \%$ White, $5 \%$ Black, 5\% Asian; 0\% Hispanic or Latino; Mage $=39.70$, SDage $=13.35$ ). The likeability study had 46 participants (53.5\% female; $79.1 \%$ White, $11.6 \%$ Black, $4.7 \%$ Asian, $2.3 \%$ Multiracial; $16.3 \%$ Hispanic or Latino; Mage $=35.53$, SDage $=12.46$ ). For each study, participants were paid $\$ 0.40$ for their participation. The average completion time was about fifteen minutes for the likeability set, six minutes for the trustworthiness set, eight minutes for the expertise set, and nine minutes for the power set.

Procedure After consenting to participate in the study, participants were told that we were interested in how informative certain descriptions were to understanding if someone had a particular characteristic. Then, for each description selected from Study 1, participants responded to the question, "When determining the extent to which someone is [POSITIVE VERSION OF CHARACTERISTIC (e.g., likeability)] versus [NEGATIVE VERSION OF CHARACTERISTIC (e.g., dislikeability)], how informative would it be to know that the person is [DESCRIPTION (e.g., helpful)]?" $(1=$ not at all informative to $8=$ extremely informative). Of course, the description changed for each question, and each participant only responded to questions about one characteristic. The 
order of descriptions was randomized for each participant. After responding to all the questions for a given characteristic, participants responded to demographic questions and were debriefed about the purpose of the study.

\section{Results}

The means and standard deviations of the informativeness ratings are reported in Tables 1, 2, 3, and 4. Across all descriptions, the average informativeness rating was $M=5.59$, with a range from 2.37 to 7.40 . As mentioned above, the purpose of this study was to determine which descriptions were most informative for each characteristic. We chose to exclude words that were rated as 5.5 or lower in informativeness. This number was chosen because generally those descriptions rated as 5.5 or higher were significantly different from the fifth point on the scale, which had the label "slightly informative," and we wanted to include those descriptions that were more than slightly informative for each characteristic. Additionally, the 5.5 cutoff left us with a reasonable number of descriptions for each characteristic: 24 likeability, 34 dislikeability, 14 powerful, 18 powerless, 25 expert, 6 inexpert, 6 trustworthy, and 15 untrustworthy descriptions (see Tables 1, 2, 3, and 4).

\section{Study 3: Comparing the characteristics}

Studies $2 \mathrm{a}-\mathrm{d}$ allowed us to identify which of the descriptions that participants generated for each characteristic were most informative. Once we had determined which descriptions were most informative for each characteristic, we wanted to examine whether those descriptions were particularly related to their corresponding characteristic compared to the others. Additionally, we wanted to examine whether the descriptions generated to capture a particular characteristic might be especially related to other characteristics to identify which potential confounds researchers would want to be aware of when manipulating each characteristic.

\section{Method}

Participants Two hundred and twenty-three Mechanical Turk workers $(63.7 \%$ female; $80.7 \%$ White, $7.2 \%$ Black, .9\% Native American, $7.2 \%$ Asian, .4\% Pacific Islander, 3.6\% Multiracial; $6.3 \%$ Hispanic or Latino; Mage $=37.72$, SDage $=13.57)$ participated in this study. Participants were paid $\$ 0.30$ for their participation, and the median completion time was roughly ten minutes.

Procedure After consenting to participate in the study, participants were told that we were interested in how informative certain descriptions were to understanding if someone has a particular characteristic. Because there were so many descriptions remaining (143), we split the descriptions into groups by the characteristic they were generated to describe. Additionally, we randomly split the likeability/dislikeability descriptions into two groups because there were so many of them. Further, we combined the trustworthy and expert descriptions into one group because there were so few of them. Then we randomly assigned participants to respond to one group of questions (either those containing the descriptions for trustworthiness and expertise $(N=61)$, power $(N=55)$, or one of the two likeability groups $(N s=58,49))$. For each description remaining after the exclusions from Study 2, participants responded to the question, "Someone you already know is providing you information on a topic. You know that this person is [DESCRIPTION (e.g., helpful)]. Given that the person is [DESCRIPTION (e.g., helpful)], please rate the extent to which the person is" $(1=$ extremely dislikeability to $6=$ extremely likeability; $1=$ extremely non-expert to $6=$ extremely expert; $1=$ extremely untrustworthy to $6=$ extremely trustworthy; and $1=$ extremely powerless to $6=$ extremely powerful). We used a revised Latin square to create four different order conditions for these trait measures. Participants were randomly assigned to respond to the trait questions in a given order across the descriptions. After responding to all the descriptions for a given characteristic, participants responded to demographic questions and were debriefed about the purpose of the study.

\section{Results and discussion}

One goal for this study was to examine whether the descriptions generated for each characteristic were better predictors of measures of that particular characteristic (trait measures) compared to measures of the other source characteristics. To test this, for each participant, we took the average of each trait measure for all of the descriptions intended to capture a particular characteristic. This allowed us to examine whether the descriptions generated for a particular characteristic better captured that characteristic than others. For each characteristic, we ran a repeated-measures analysis of variance (ANOVA) examining an interaction between the valence factor (with two levels: positive vs. negative) and the trait measure factor (with four levels corresponding to each of the four source characteristics: likeability, trustworthiness, expertise, and power). For each of these analyses, we used a multivariate test, which is valid even without sphericity or balanced data, which are requirements for univariate test validity. For each of our analyses, various multivariate tests (Pillai's trace, Wilks' lambda, Hotelling's trace, and Roy's largest root) showed the same results.

Relative influences on perceptions of "intended" versus "unintended" traits Although there were two sets of likeability descriptions that participants responded to, we present 
analyses collapsed across the two sets here. The set did moderate the key interaction of interest $(p=.009)$. However, this interaction reflected that each study set significantly showed the same patterns of results, but to different degrees. The results for each individual set support the same conclusions presented here and are available in the Online Supplement for interested readers. Among those descriptions generated for the (dis)likeability descriptions, the key interaction between valence and the trait measures was significant, $F(3$, $104)=130.18, p<.0001, \eta_{\mathrm{p}}{ }^{2}=.79$ (Fig. 1). To test whether this interaction was due to the difference between the positive and negative descriptions better predicting the difference between likeability and dislikeability more so than each of the other traits, we ran three two-way mixed general linear models (GLMs) comparing likeability to each of the other characteristics. The difference between the positive and the negative descriptions was greater for the ratings of likeability $(M=$ $5.34, S D=.54$ vs. $M=1.68, S D=.59)$ than trustworthiness $(M=4.93, S D=.62$ vs. $M=2.10, S D=.63 ;$ Valence $\times$ Characteristic Rating, $F(1,106)=174.34, p<.001$, $\left.\eta_{\mathrm{p}}{ }^{2}=.62\right)$, expertise $(M=4.25, S D=.73$ vs. $M=2.68$, $S D=.79$; Valence $\times$ Characteristic Rating, $F(1,106)=$ $\left.316.85, p<.001, \eta_{\mathrm{p}}{ }^{2}=.75\right)$, or power $(M=4.19, S D=.76$ vs. $M=3.11, S D=.95 ; F(1,106)=342.22, p<.001$, $\eta_{\mathrm{p}}{ }^{2}=.76$ ), indicating that the descriptions generated to describe likeability did indeed predict likeability better than the other characteristics. There were also significant main effects of the trait measures, $F(3,104)=8.95, p<.001, \eta_{\mathrm{p}}{ }^{2}=.21$, and valence, $F(1,106)=635.50, p<.0001, \eta_{\mathrm{p}}{ }^{2}=.86$.

Among those descriptions generated for (un)trustworthiness, the key interaction between valence and the trait measures was significant, $F(3,58)=42.30, p<.001$, $\eta_{\mathrm{p}}{ }^{2}=.69$ (Fig. 2). To test whether this interaction was due to the difference between the positive and negative descriptions better predicting the difference between trustworthiness and untrustworthiness more so than each of the other characteristics, we ran three two-way mixed GLMs comparing trustworthiness ratings to ratings of each of the other traits. The difference between the positive and the negative descriptions was greater for ratings of trustworthiness $(M=5.29, S D=.58$ vs. $M=1.52, S D=.54)$ than for ratings of likeability $(M=5.12$, $S D=.53$ vs. $M=1.80, S D=.69$; Valence $\times$ Characteristic Rating, $\left.F(1,60)=36.16, p<.001, \eta_{\mathrm{p}}{ }^{2}=.38\right)$, expertise $(M=$ $4.43, S D=.62$ vs. $M=2.27, S D=.91$; Valence $\times$ Characteristic Rating, $\left.F(1,60)=105.17, p<.001, \eta_{\mathrm{p}}{ }^{2}=.64\right)$, and power $(M=4.40, S D=.62$ vs. $M=2.58, S D=.98$; Valence $\times$ Characteristic Rating, $F(1,60)=128.01, p<.001$, $\eta_{\mathrm{p}}{ }^{2}=.68$ ), indicating that the descriptions generated to describe trustworthiness did indeed predict trustworthiness better than the other characteristics. There was also a significant main effect of the trait measure, $F(3,58)=6.06, p=.001$, $\eta_{\mathrm{p}}{ }^{2}=.24$, and of the valence factor, $F(1,60)=455.04$, $p<.001, \eta_{\mathrm{p}}^{2}=.88$.

Among those descriptions generated for (in)expertise, the key interaction between valence and the trait measures was significant, $F(3,58)=47.61 p<.001, \eta_{\mathrm{p}}^{2}=.71$ (Fig. 3). To test whether this interaction was due to the difference between the positive and negative descriptions better predicting the difference between expertise and lack of expertise than the other traits, we ran three two-way mixed GLMs comparing expertise ratings to each of the other characteristics. The difference between the positive and the negative descriptions was greater for ratings of expertise $(M=4.88, S D=.56$ vs. $M=$ $1.64, S D=.64)$ than for ratings of likeability $(M=4.56$, $S D=.58$ vs. $M=2.86, S D=.88$; Valence $\times$ Characteristic Rating, $\left.F(1,60)=145.20, p<.001, \eta_{\mathrm{p}}{ }^{2}=.71\right)$,

Effect of Liking Descriptions on Each Characteristic

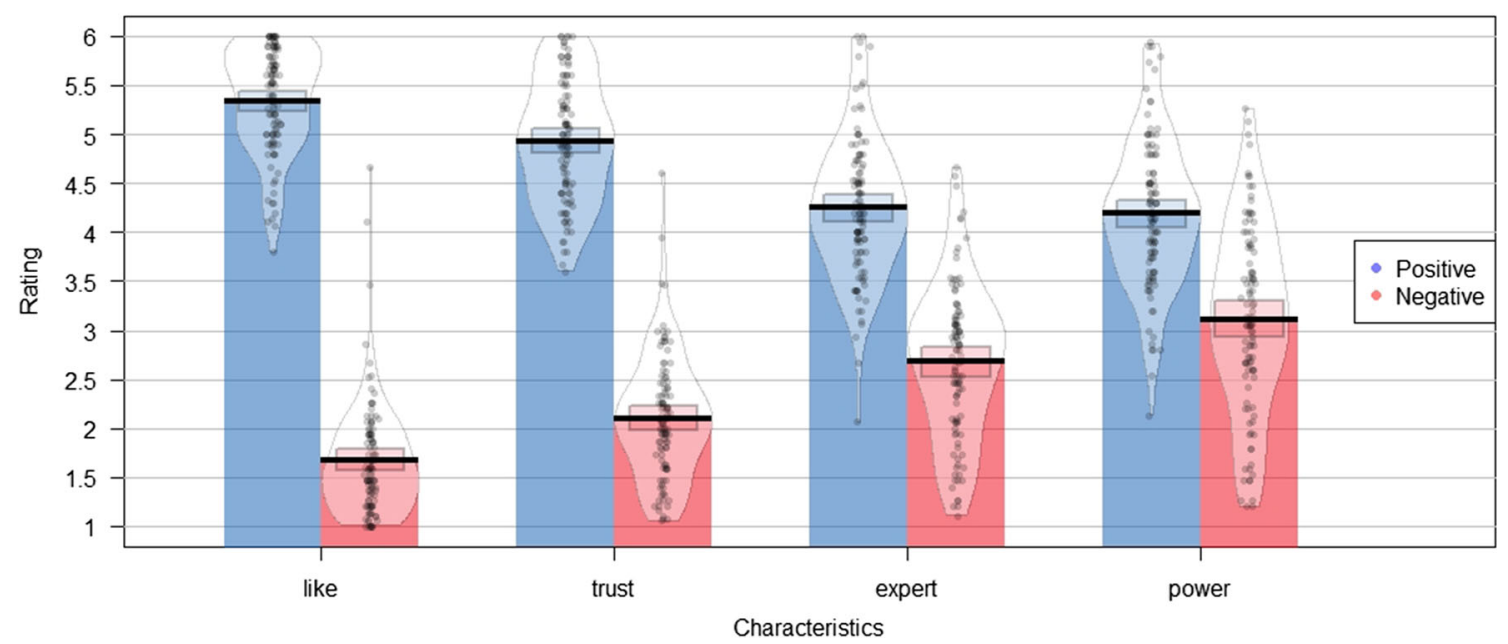

Fig. 1 RDI (raw (data), description, \& infererence) plot for the effect of the positive and negative likeability descriptions on each of the

of each condition. The box around the dark line represents the $95 \%$ confidence interval. The smooth density curve shows the data distribution 
Effect of Trustworthiness Descriptions on Each Characteristic

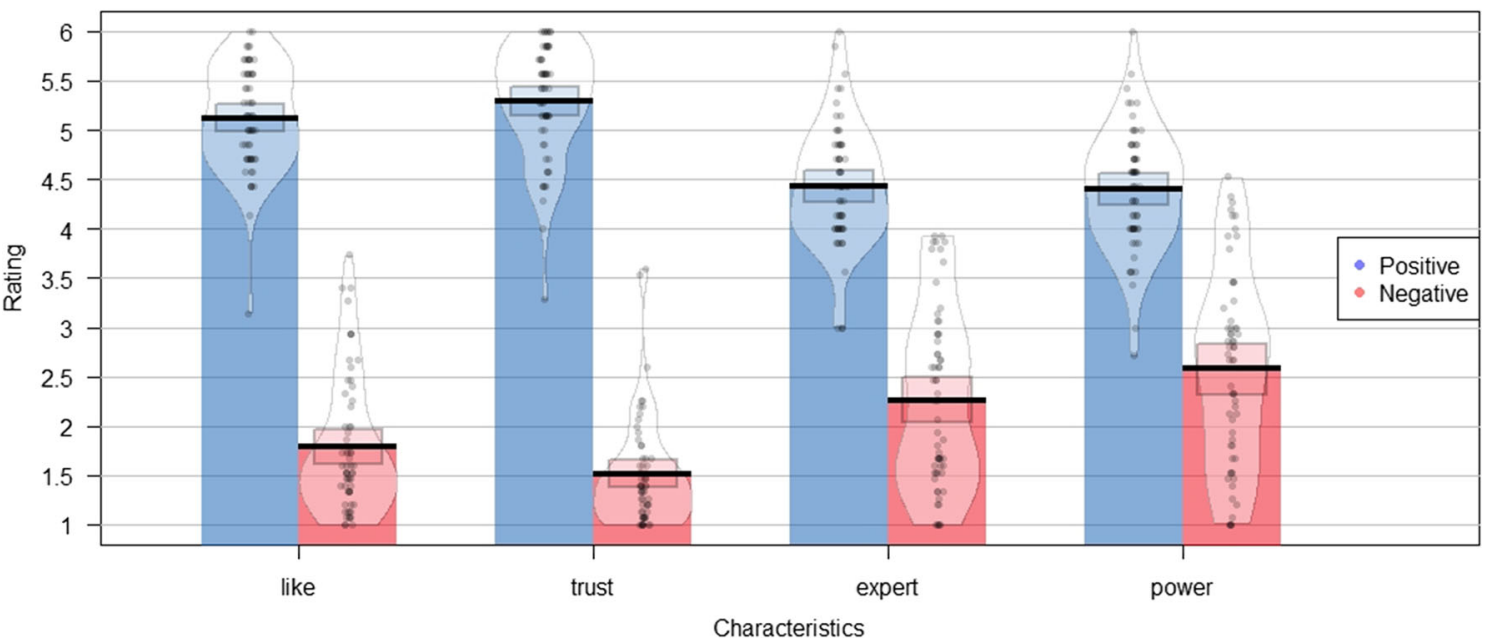

Fig. 2 RDI plot for the effect of the positive and negative trustworthiness descriptions on each of the characteristics in Study 3. The dark horizontal line indicates the mean of each condition. The box around the dark line represents the $95 \%$ confidence interval. The smooth density curve shows the data distribution

test whether this interaction was due to the difference between the positive and negative descriptions better predicting the difference between powerful and powerless more so than the other characteristics, we ran three two-way mixed GLMs comparing power ratings to each of the other characteristics. The difference between the positive and the negative descriptions was greater for ratings of power $(M=4.72, S D=.73$ vs. $M=1.94, S D=.73)$ than for ratings of likeability $(M=3.78$, $S D=.72$ vs. $M=3.01, S D=.67$; Valence $\times$ Characteristic Rating, $\left.F(1,54)=118.14, p<.001, \eta_{\mathrm{p}}{ }^{2}=.69\right)$, trustworthiness $(M=3.71, S D=.72$ vs. $M=2.87, S D=.69$; Valence $\times$ Characteristic Rating, $\left.F(1,54)=135.37, p<.001, \eta_{\mathrm{p}}{ }^{2}=.72\right)$, and expertise $(M=4.16, S D=.71$ vs. $M=2.50, S D=.65$; significant, $F(3,52)=43.45, p<.001, \eta^{2}=72($ Fig. 4). To

Effect of Expert Descriptions on Each Characteristic

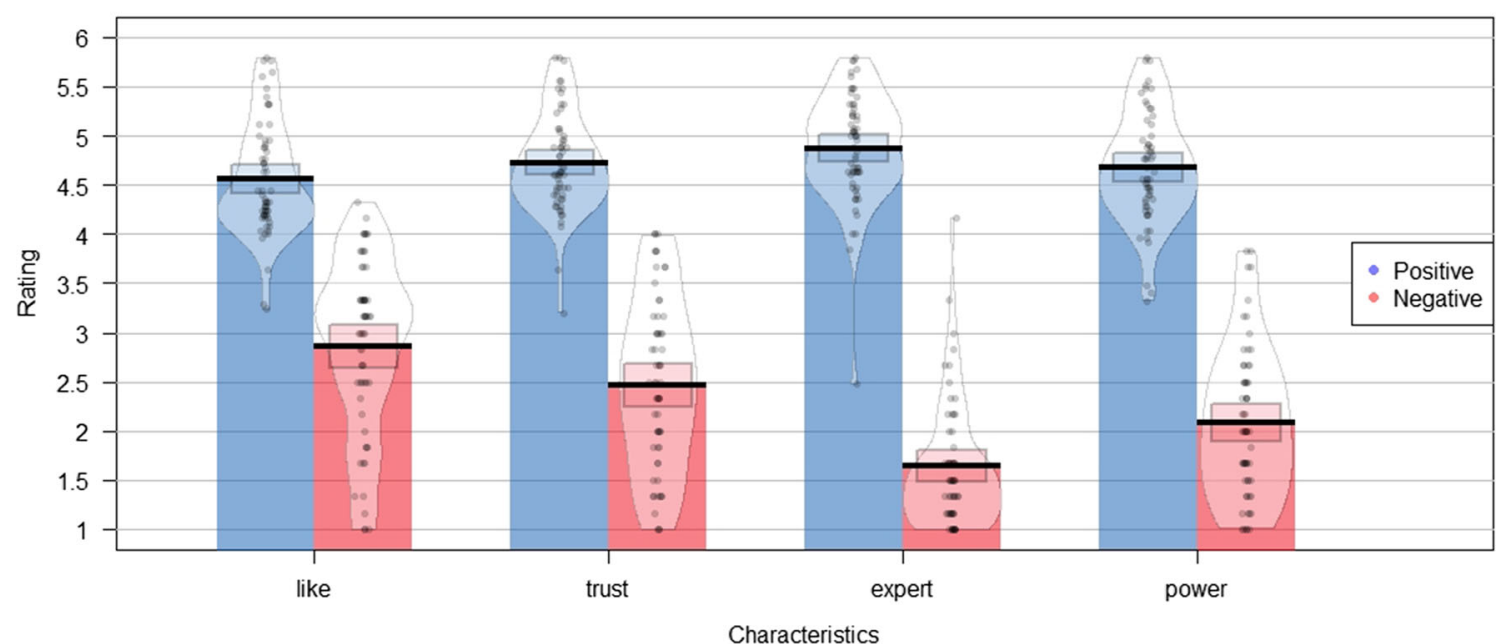

Fig. 3 RDI plot for the effect of the positive and negative expertise descriptions on each of the characteristics in Study 3. The dark horizontal line indicates the mean of each condition. The box around the dark line represents the $95 \%$ confidence interval. The smooth density curve shows the data distribution 


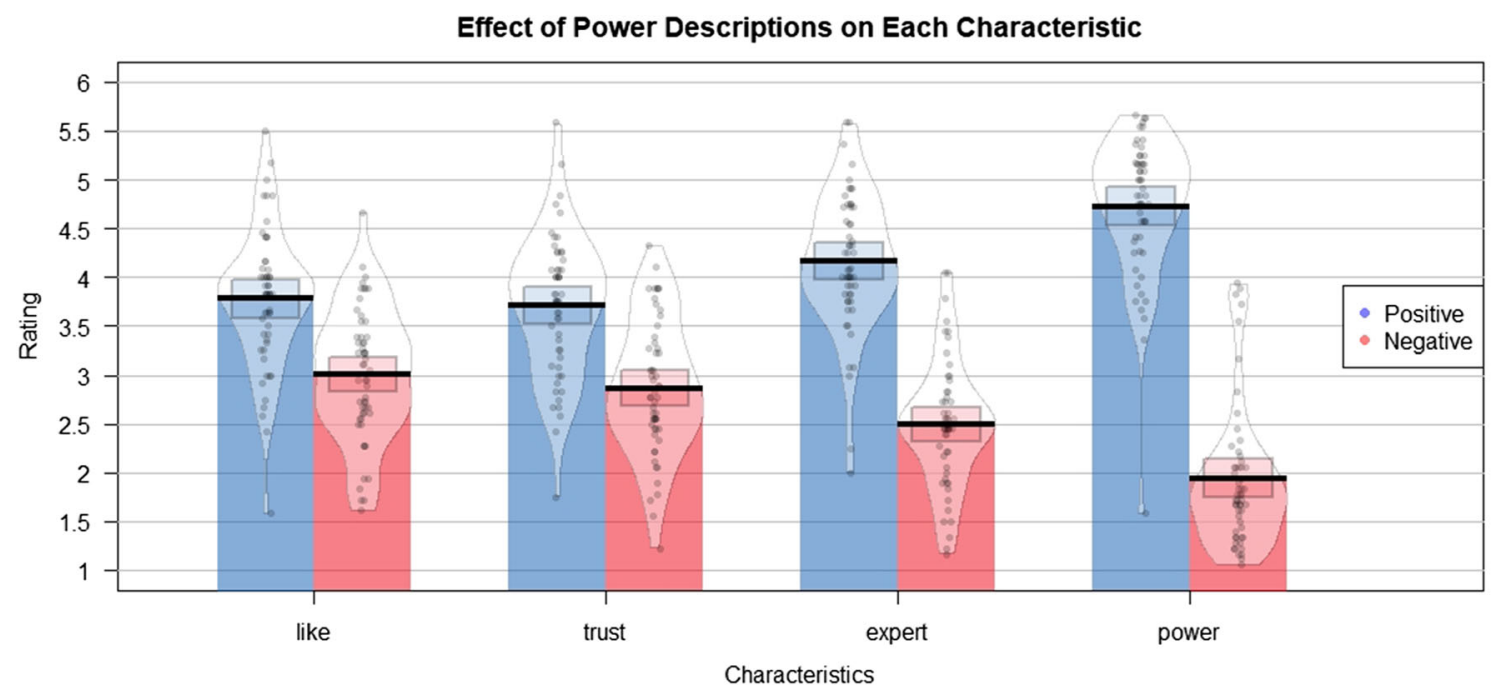

Fig. 4 RDI plot for the effect of the positive and negative power descriptions on each of the characteristics in Study 3 . The dark horizontal line indicates the mean of each condition. The box around the dark line represents the $95 \%$ confidence interval. The smooth density curve shows the data distribution.

Valence $\times$ Characteristic Rating, $F(1,54)=90.73, p<.001$, $\eta_{\mathrm{p}}^{2}=.63$ ), indicating that the descriptions generated to describe power did indeed predict power better than the other characteristics. The main effect of the trait measures was significant, $F(3,52)=2.89, p=.04, \eta_{\mathrm{p}}{ }^{2}=.14$, and the main effect of valence was significant, $F(1,54)=148.27, p<.001$, $\eta_{\mathrm{p}}{ }^{2}=.73$.

Identifying the most likely confounds across traits In addition to providing insight about whether the descriptions intended to describe a particular characteristic did so better than other traits, we also wanted to identify potential confounds about which researchers would need to be particularly concerned if creating manipulations based on these lay conceptions.

In particular, the difference that the (dis)likeability descriptions created in trustworthiness was larger than expertise, $F(1,106)=154.19, p<.001, \eta_{\mathrm{p}}{ }^{2}=.59$, or power, $F(1,106)=175.67, p<.001, \eta_{\mathrm{p}}^{2}=.62$, in both sets of liking descriptions. These stronger relations between likeability and trustworthiness are consistent with work on the stereotype content model that identifies warmth and competence as two fundamental dimensions of person perception (Cuddy, Fiske, \& Glick, 2008). In that context, both likeability (sociability) and trustworthiness (morality) have been identified as part of warmth (e.g., Landy, Piazza, \& Goodwin, 2016; Leach, Ellemers, \& Barreto, 2007). Consistent with these findings, the valence of the trustworthiness descriptions also had a larger effect on rated source likeability than on expertise, $F(1$, $60)=72.23, p<.001, \eta_{\mathrm{p}}{ }^{2}=.55$, or power, $F(1,60)=$ 92.36, $p<.001, \eta_{\mathrm{p}}{ }^{2}=.61$. In addition, the difference on expertise was larger than on power, $F(1,60)=12.40$, $p=.001, \eta_{\mathrm{p}}{ }^{2}=.17$. This stronger relation between trustworthiness and expertise might reflect that they represent two components of source credibility (Hovland et al., 1953). Additionally, this would be consistent with work demonstrating that people make inferences about how competent someone is based on their moral behavior (Stellar \& Willer, 2018). Parallel results also appear when examining effects of the expertise descriptions on the other characteristics. The valence of the expertise descriptions had larger effects on trustworthiness than liking, $F(1,60)=32.88, p<.001, \eta_{\mathrm{p}}{ }^{2}=.35$.

Additionally, the valence of the expertise descriptions had larger effects on power than liking, $F(1,60)=44.77, p<.001$, $\eta_{\mathrm{p}}{ }^{2}=.43$, and, though to a lesser extent, larger effects on power than trustworthiness, $F(1,60)=7.01, p=.01, \eta_{\mathrm{p}}{ }^{2}=.11$. This pattern could reflect that expertise and power each contribute to an overall perception of competence or agency. Analyses examining the effect of the valence of the power descriptions support this general idea, with the power descriptions having a larger effect on expertise than either trustworthiness, $F(1,54)=70.51, p<.001, \eta_{\mathrm{p}}{ }^{2}=.57$, or liking, $F(1$, $54)=66.06, p<.001, \eta_{\mathrm{p}}{ }^{2}=.55$. There was no difference in the effects of the power descriptions on trustworthiness versus liking, $F(1,54)=1.29, p=.26, \eta_{\mathrm{p}}{ }^{2}=.02$.

Based on these analyses, it seems that trustworthiness and likeability manipulations are particularly likely to affect one another, which makes sense given that they would both fall into the "warmth" dimension of person perception (Cuddy et al., 2008; see Leach et al., 2007, for recent work splitting warmth into "sociability" and "morality"). It is also possible that expertise and power are particularly likely to affect one another, perhaps reflecting different components of competence or agency (Koch, Imhoff, Dotsch, Unkelbach, \& Alves, 2016). Given the relations between 
trustworthiness and likeability as well as expertise and power, researchers might want to be particularly thoughtful about whether a given influence of trustworthiness (or expertise) might partly be due to likeability (or power) or vice versa. It is worth noting that the descriptions generated for each trait affect each of the unintended characteristics, $p<.001$, as well. So, in general, researchers may want to consider influences on each of these variables, but the current analysis provides some insight into which traits might be most likely to be affected. Correlations between each of the variables within each characteristic set and within the positive and negative description sets also support this general pattern of relations (Table 5). In some settings, whether effects are due to one source variable or the other might not be the key question, but in other cases, the distinctions between the particular traits might constitute the very nature of the research claim. In either case, however, it seems wise, given the current results, to be aware of which alternative source traits might also play a role when intending to manipulate a particular aspect of the source.

The above analyses are based on the means of all the most prototypical descriptions of each source characteristic.
However, there are likely some individual descriptions that uniquely affect their intended characteristic more than others. Thus, readers may want more detailed information about how each individual description was rated for each of the traits. By examining each description, researchers could potentially identify particular descriptors that are not as associated with other source characteristics. The supplemental materials contain graphs intended to illustrate these. The descriptions generated for each characteristic are graphed along the ratings for each possible combination of traits. Furthermore, there is a table with the trait ratings for each description in the supplemental materials. These resources would be particularly helpful to those interested in which descriptions more uniquely relate to one characteristic or another versus which capture some characteristics relatively equally. For example, if researchers were looking to have a purer manipulation of liking, they might use "fun" rather than "caring" to avoid a confound with trustworthiness. Similarly, when manipulating inexpertise, researchers might want to use "untrained" rather than "stupid" to avoid a confound with liking, trustworthiness, and power.

Table 5 Correlations between liking, perceived trustworthiness, perceived expertise, and perceived power in each characteristic set, split by the positive and the negative descriptions

\begin{tabular}{|c|c|c|c|c|c|c|}
\hline & \multicolumn{2}{|l|}{ Like } & \multicolumn{2}{|l|}{ Trust } & \multicolumn{2}{|l|}{ Expert } \\
\hline & Positive Set & Negative Set & Positive Set & Negative Set & Positive Set & Negative Set \\
\hline \multicolumn{7}{|c|}{ Liking Set } \\
\hline \multicolumn{7}{|l|}{ Like } \\
\hline Trust & $.75 * * *$ & $.80^{* * *}$ & & & & \\
\hline Expert & $.37 * * *$ & $.47 * * *$ & $.55^{* * *}$ & $.60 * * *$ & & \\
\hline Power & $.31 * *$ & $.30 * *$ & $.37 * * *$ & $.42 * * *$ & $.81 * * *$ & $.76^{* * *}$ \\
\hline \multicolumn{7}{|c|}{ Trustworthiness Set } \\
\hline \multicolumn{7}{|l|}{ Like } \\
\hline Trust & $.73 * * *$ & $.86^{* * *}$ & & & & \\
\hline Expert & $.37 * *$ & $.64 * * *$ & $.42 * *$ & $.49 * * *$ & & \\
\hline Power & $.35^{* *}$ & $.60 * * *$ & $.41 * *$ & $.44 * * *$ & $.77 * * *$ & $.87 * * *$ \\
\hline \multicolumn{7}{|c|}{ Expertise Set } \\
\hline \multicolumn{7}{|l|}{ Like } \\
\hline Trust & $.78 * * *$ & $.75 * * *$ & & & & \\
\hline Expert & $.58 * * *$ & $.46^{* * * *}$ & $.70 * * *$ & $.54 * * *$ & & \\
\hline Power & $.60 * * *$ & $.54 * *$ & $.72 * * *$ & $.59 * * *$ & $.73 * * *$ & $.76^{* * * *}$ \\
\hline \multicolumn{7}{|c|}{ Power Set } \\
\hline \multicolumn{7}{|l|}{ Like } \\
\hline Trust & $.81 * * *$ & $.84 * * *$ & & & & \\
\hline Expert & $.67 * * *$ & $.61 * * *$ & $.71 * * *$ & $.73 * * *$ & & \\
\hline Power & $.25 * *$ & $.40 * *$ & $.31 * * *$ & $.63 * * *$ & $.55 * * *$ & $.75 * * *$ \\
\hline
\end{tabular}

$* p<.05 . * * p<.01 . * * * p<.001$ 


\section{General discussion}

The current paper provided a prototype analysis of four prominent source characteristics: likeability, trustworthiness, expertise, and power. In Study 1, participants generated descriptions for each characteristic. Study 2 allowed us to determine which descriptions were perceived as most informative of a source's standing on each characteristic. Finally, Study 3 demonstrated that the generated descriptions for each source characteristic were more predictive of differences in their intended characteristic than the other characteristics, providing support that the descriptions for each characteristic captured a relatively unique prototype. However, Study 3 also makes clear that lay conceptions of the various source characteristics also show substantial overlap in that a description intended to represent the positive versus negative poles of any one source characteristic also created variation in the other source characteristics (though less than for the intended characteristic). Such overlap might be expected given general halo effects in person impressions (for review, see Cooper, 1981). This issue would not necessarily be confined to prototype-based manipulations of source characteristics, but could also be important to consider when using a particular person or set of "qualifications" to manipulate characteristics of the source (e.g., describing the source's education if manipulating expertise or providing information about previous behaviors of a likeability versus dislikeability source). Most such manipulations of a source characteristic would be intended to compare two or more conditions that cover much of the continuum along which that characteristic lies. Therefore, it might often be the case that individual sources would be as discrepant as the descriptors generated in the current research for the likeability/dislikeability, trustworthy/untrustworthy, expert/inexpert, or powerful/powerless sources. In so doing, however, researchers should be aware that intended differences in one dimension might also be creating unintended differences in another dimension(s), potentially creating a construct validity threat. In particular, ratings of trustworthiness and likeability were strongly related, and ratings of expertise and power were particularly related (though previous research has also shown that perceived expertise of a source can definitely be distinguished from the source's ability to implement an advocated action, which would seem to also be a key part of power; see Clark \& Wegener, 2009). Ultimately, our hope is that this work provides a methodological tool to understand how people perceive source characteristics along the dimensions that are the most prominently used in persuasion research (and in other related domains). The rating values for individual source descriptors could help address construct validity threats. They may be particularly useful for researchers who would like to create manipulations of the source characteristics that remain relatively constant across time and situations (rather than relying on different source exemplars for different domains and, potentially, different time periods).

The interrelations among the source characteristics also suggest the possibility that previous manipulations of any one of these characteristics might have inadvertently affected one or more of the other characteristics. To the extent that has occurred, it might become unclear whether the original findings were attributable solely to the characteristic the researchers intended to manipulate. In some cases, that might not pose much of a conceptual problem, as the source characteristics might be relatively interchangeable, as in basic science demonstrations of source characteristics serving as a peripheral cue to guide persuasion when ability or motivation to think about the content of the persuasive message is lacking (e.g., Petty et al., 1981, 1983). In other cases, however, theory might suggest that a particular source characteristic is important in the effect. For example, if part of a processing effect is thought to be due to the threat involved in a counter-attitudinal persuasive message, it may be important that the message source is perceived as an expert or as otherwise effective in implementing advocated actions (e.g., Clark \& Wegener, 2009; Clark, Wegener, Habashi, \& Evans, 2012; Clark, Wegener, Sawicki, Petty, \& Briñol, 2013). The same theory might not be equally applicable to sources simply perceived as likeability (though some recent research suggests that sociability/likeability can be viewed as helping to determine how effective the person will be in recruiting others to support their intentions, Landy et al., 2016). When a particular aspect of the source is key to the proposed psychological mechanism, it would be more important to be sure that one is only manipulating the intended characteristic. It could be that many possible manipulations will affect more than one source characteristic, such that researchers might need to control for influences on other potential characteristics either through measurement of the various perceived source characteristics or by triangulation across different manipulations that might differ in the "irrelevancies" that go along with each manipulation (Cook \& Campbell, 1979). For example, it may be nearly impossible to manipulate trustworthiness without affecting likeability (Landy et al., 2016). However, it may be possible to manipulate likeability without affecting trustworthiness (perhaps through descriptions like "fun" and "easy-going"). A researcher interested in the effects of trustworthiness per se might include a condition manipulating likeability but not trustworthiness to examine whether there are unique effects of trustworthiness-imbued likeability beyond any effects of likeability per se.

One potential limitation of this work is that we did not have participants identify descriptions that were uniquely one of the characteristics (e.g., trustworthy, but not likeability). As can 
be seen in the graphs in the supplemental materials, many of the descriptions have effects on several of the characteristics. It could be that such "unique" descriptions are not as prototypical in perceivers' minds, but they could nonetheless be very useful for psychological research. It would be useful for future work to identify descriptions perceived as uniquely capturing one of the characteristics to provide researchers with manipulations that would only affect their variable of interest. It seems likely that the descriptions generated in that type of study would be less central or prototypical to each characteristic but would allow for experimentally isolating effects of characteristics.

An additional limitation concerns our use of convenience samples. To collect data for these studies, we relied on samples from Amazon's Mechanical Turk. This approach seemed useful and appropriate because many current persuasion researchers use Mechanical Turk for data collection. Further, this resulted in a more diverse sample than could have been obtained using only participants at our university. However, the current data certainly do not make up a representative sample. Additionally, although the current sample sizes sufficiently powered the key within-subject analyses of interest, they only allow limited examination of moderation by participant characteristics. We did conduct some exploratory tests of moderation by age and gender in Studies 2 and 3. We observed some small differences in how men and women rated informativeness in Study 2. Both men and women showed the same valence $\times$ trait patterns, but sometimes to different degrees. There were also some age-based differences in how people rated informativeness in Study 2. These primarily reflected that younger people view prejudice as more informative of liking than older people do, and some age-based dialect differences. Once again, these differences occurred mostly in the strength of the same overall patterns of data across ages. Gender did not significantly moderate any analyses in Study 3 , but age did. However, this moderation again reflected different size effects that were in the same direction and significant among both older and younger people. Therefore, we do not find these differences to be particularly meaningful. These moderation analyses are available in the Online Supplement for readers who may be interested. It is possible that different people would have different conceptions of these characteristics, so if researchers wanted to use a population very different from the one we used, we would recommend that researchers pre-test these suggested source descriptions in their population.

Our prototype analysis of the core source characteristics of likeability, trustworthiness, expertise, and power provides insight into the perceptions that people have of each type of source. For example, if a perceiver is simply told by another person that a source is trustworthy, the current generated and tested descriptors might represent a good picture of what the perceiver comes to believe about the source (or at least what they believe is being communicated by the trustworthy label). Ultimately, our hope is that this new methodological tool will help address construct validity concerns in source characteristic research; these prototypes will help researchers to gain a better understanding of how sources in their studies are perceived and to develop more effective manipulations of the source characteristics they wish to study.

Acknowledgements This work was supported by the National Science Foundation Graduate Research Fellowship Program (DGE-1343012).

Open practices statement The data for all experiments are available at https://osf.io/4e6hy/?view_only=e28832c0a5bf4ed38671579070461fd3. Further, the design of these studies was extremely simple, so the exact materials should be apparent from the methods descriptions, but we would be happy to provide an alternative stimulus file if that seemed necessary. None of these studies were preregistered.

\section{Compliance with ethical standards}

Conflicts of interest The authors have no conflicts of interest to declare.

\section{References}

Abelson, R. P., \& Miller, J. C. (1967). Negative persuasion via personal insult. Journal of Experimental Social Psychology, 3(4), 321-333.

Chaiken, S. (1986). Physical appearance and social influence. In C. P. Herman, M. P. Zanna, \& E. T. Higgins (Eds.), Physical appearance, stigma, and social behavior: The Ontario Symposium (3rd). Hillsdale, NJ: Erlbaum.

Chaiken, S., Liberman, A., \& Eagly, A. H. (1989). Heuristic and systematic information processing within and beyond the persuasion context. In James S. Uleman \& John A. Bargh (Eds.), Unintended thought (pp. 212-252). New York, NY, US: Guilford Press.

Clark, J. K., \& Wegener, D. T. (2009). Source entitativity and the elaboration of persuasive messages: The roles of source efficacy and message discrepancy. Journal of Personality and Social Psychology, 97, 42-57.

Clark, J. K., Wegener, D. T., Habashi, M. M., \& Evans, A. T. (2012). Source expertise and persuasion. Personality and Social Psychology Bulletin, 38(1), 90-100.

Clark, J. K., Wegener, D. T., Sawicki, V., Petty, R. E., \& Briñol, P. (2013). Evaluating the message or the messenger? Implications for self-validation in Persuasion. Personality and Social Psychology Bulletin, 39(12), 1571-1584.

Cook, T. D., \& Campbell, D. T. (1979). Quasi-experimentation: Design and analysis issues for field settings. Chicago, IL: Rand McNally College Publishing Company.

Cooper, W. H. (1981). Ubiquitous halo. Psychological Bulletin, 90(2), 218-244.

Cuddy, A. J. C., Fiske, S. T., \& Glick, P. (2008). Warmth and competence as universal dimensions of social perception: The stereotype content model and the BIAS Map. In Mark P. Zanna (Ed.), Advances in Experimental Social Psychology (Vol. 40, pp. 61-149). San Diego, CA: Elsevier Academic Press.

Hovland, C. I., Janis, I. L., \& Kelley, H. H. (1953). Communication and persuasion: Psychological studies of opinion change. New Haven, CT: Yale University Press.

Kelman, H. C. (1958). Compliance, identification, and internalization three processes of attitude change. Journal of Conflict Resolution, 2(1), 51-60. 
Kelman, H. C., \& Hovland, C. I. (1953). "Reinstatement" of the communicator in delayed measurement of opinion change. The Journal of Abnormal and Social Psychology, 48(3), 327-335.

Koch, A., Imhoff, R., Dotsch, R., Unkelbach, C., \& Alves, H. (2016). The $\mathrm{ABC}$ of stereotypes about groups: Agency/socioeconomic success, conservative-progressive beliefs, and communion. Journal of Personality and Social Psychology, 110(5), 675-709.

Landy, J. F., Piazza, J., \& Goodwin, G. P. (2016). When it's bad to be friendly and smart. Personality and Social Psychology Bulletin, 42(9), 1272-1290.

Leach, C. W., Ellemers, N., \& Barreto, M. (2007). Group virtue: The importance of morality (vs. competence and sociability) in the positive evaluation of in-groups. Journal of Personality and Social Psychology, 93(2), 234-249.

Petty, R. E., \& Cacioppo, J. T. (1986). Communication and persuasion: Central and peripheral routes to attitude change. New York: Springer/Verlag.

Petty, R. E., Cacioppo, J. T., \& Goldman, R. (1981). Personal involvement as a determinant of argument- based persuasion. Journal of Personality and Social Psychology, 41, 847-855.

Petty, R. E., Cacioppo, J. T., \& Schumann, D. (1983). Central and peripheral routes to advertising effectiveness: The moderating role of involvement. Journal of Consumer Research, 10(2), 135.

Petty, R. E., Wegener, D. T., \& White, P. H. (1998). Flexible correction processes in social judgment: Implications for persuasion. Social Cognition, 16(1), 93-113.
Priester, J. R., \& Petty, R. E. (2003). The influence of spokesperson trustworthiness on message elaboration, attitude strength, and advertising effectiveness. Journal of Consumer Psychology, 13(4), 408421.

Snyder, M., \& Rothbart, M. (1971). Communicator attractiveness and opinion change. Canadian Journal of Behavioural Science/Revue Canadienne Des Sciences Du Comportement, 3(4), 377-387.

Stellar, J. E., \& Willer, R. (2018). Unethical and inept? The influence of moral information on perceptions of competence. Journal of Personality and Social Psychology, 114(2), 195-210.

Wallace, L. E., Wegener, D. T., \& Petty, R. E. (2020a). When Sources Honestly Provide Their Biased Opinion: Bias as a Distinct Source Perception With Independent Effects on Credibility and Persuasion. Personality and Social Psychology Bulletin, 46(3), 439-453.

Wallace, L. E., Wegener, D. T., \& Petty, R. E. (2020b). Influences of Source Bias That Differ From Source Untrustworthiness: When Flip-Flopping Is More and Less Surprising. Journal of Personality and Social Psychology, 118(4), 603-616.

Zimbardo, P. G., Weisenberg, M., Firestone, I., \& Levy, B. (1965). Communicator effectiveness in producing public conformity and private attitude change. Journal of Personality, 33(2), 233-255.

Publisher's note Springer Nature remains neutral with regard to jurisdictional claims in published maps and institutional affiliations. 